\title{
Direct Catalytic Asymmetric Aldol-Type Reaction of Aldehydes with Ethyl Diazoacetate
}

\author{
Wengang Yao, Jianbo Wang*
}

Key Laboratory of Bioorganic Chemistry and Molecular Engineering of Ministry of Education, Department of Chemical Biology, College of Chemistry, Peking University, Beijing 100871, China

wangjb@pku.edu.cn

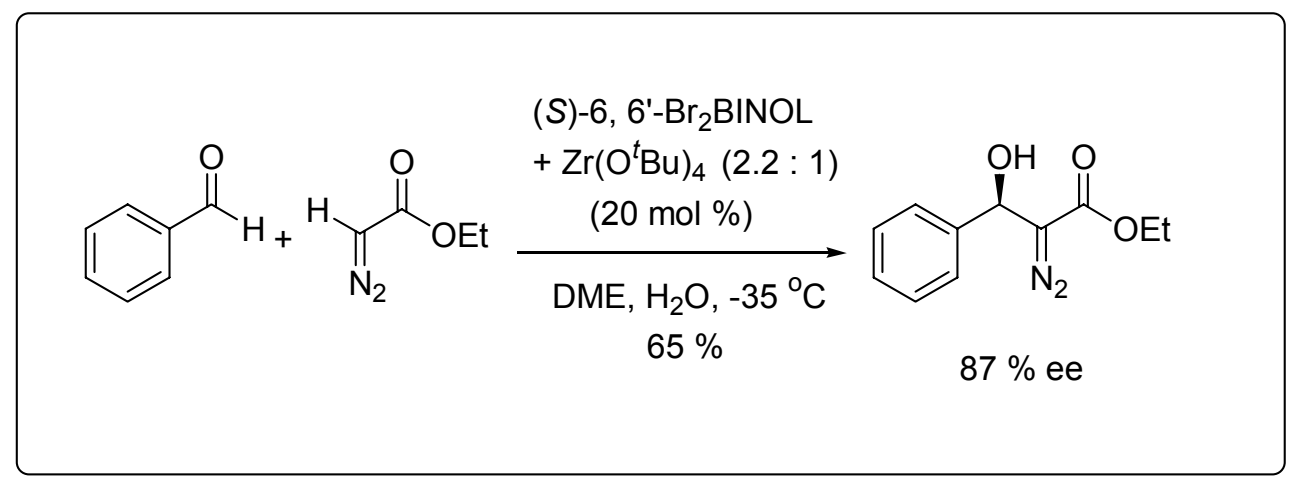


General. All reactions were performed under a nitrogen atmosphere in a flame-dried reaction flask, and the components were added via Syringe. All solvents were distilled prior to use. For chromatography, 100-200 mesh silica gel (Qindao, China) was employed. ${ }^{1} \mathrm{H}$ and ${ }^{13} \mathrm{C}$ NMR spectra were recorded at 300 $\mathrm{MHz}$ and $75 \mathrm{MHz}$ with Varian Mercury 300 spectrometer. Chemical shifts are reported in ppm using tetramethylsilane as internal standard. IR spectra were recorded with a Nicolet 5MX-S infrared spectrometer. Mass spectra were obtained on a VG ZAB-HS mass spectrometer. $\operatorname{Zr}\left(\mathrm{O}^{t} \mathrm{Bu}\right)_{4}$ was purchased from Fluka. HPLC analysis was performed at HP 1100 apparatus with Chiralcel OJ column.

Typical procedure of the catalytic asymmetric condensation: Chiral ligand $(0.056 \mathrm{mmol})$ was dissolved in $0.5 \mathrm{~mL}$ of anhydrous DME, and then was added $\mathrm{Zr}\left(\mathrm{O}^{t} \mathrm{Bu}\right)_{4}(97 \%, 10 \mathrm{mg}, 0.025 \mathrm{mmol})$ to the solution under $\mathrm{N}_{2}$ at r.t.. After stirred for 1 hour, $\mathrm{N}_{2} \mathrm{CHCO}_{2} \mathrm{Et}(43 \mathrm{mg}, 0.375 \mathrm{mmol}$ ) was added to the solution, and then water $(0.45 \mathrm{uL}, 0.025 \mathrm{mmol})$ was added. After the solution was stirred for another $3 \mathrm{~h}$, it was cooled by dry ice $/ \mathrm{CCl}_{4}$ bath $\left(-23^{\circ} \mathrm{C}\right)$ or dry ice $/ \mathrm{ClCH}_{2} \mathrm{CH}_{2} \mathrm{Cl}$ bath $\left(-35^{\circ} \mathrm{C}\right)$. Aldehyde $(0.125 \mathrm{mmol})$ was added under $\mathrm{N}_{2}$. The solution was stirred for 3 days. And the solvent and excess $\mathrm{N}_{2} \mathrm{CHCO}_{2}$ Et were removed with rotvap. The crude residue was purified with silica gel column (petroleum ether/acetone $=$ $8: 1)$.

In entries $3,4,7$ and 8 of Table $2, \operatorname{MgBr}_{2}(0.188 \mathrm{mmol})$ was added before the addition of the aldehyde.

In the cases of 3a and the entries $2,3,4$ of Table 2 , the crude $\beta$-hydroxyl product was directly converted into $\beta$-acetoxy product by the following procedure: To the crude $\beta$-hydroxyl product was added $\mathrm{CH}_{2} \mathrm{Cl}_{2}(5 \mathrm{~mL})$, triethylamine $(3 \mathrm{~mL})$, DMAP $(5 \mathrm{mg})$ and acetic anhydride $(1 \mathrm{~mL})$. The solution was stirred overnight between $0{ }^{\circ} \mathrm{C}$ and room temp. Saturated aqueous $\mathrm{NaHCO}_{3}(20 \mathrm{~mL})$ was added, and the mixture was extracted with $\mathrm{CH}_{2} \mathrm{Cl}_{2}$ for 3 times. The combined extracts were dried over $\mathrm{Na}_{2} \mathrm{SO}_{4}$. The solvent was removed in vacuum, and the residue was purified with silica gel column (petroleum ether : acetone $=8: 1)$.

Ethyl 2-Diazo-3-phenyl-3-hydroxypropanoate $3 a .87 \%$ ee. $[\alpha]_{\mathrm{D}}{ }^{20}=-9.5\left(\mathrm{c} 3.2, \mathrm{CH}_{2} \mathrm{Cl}_{2}\right) ;{ }^{1} \mathrm{H} \mathrm{NMR}$ $\left(\mathrm{CDCl}_{3}\right) \delta 1.29(\mathrm{t}, J=7.2 \mathrm{~Hz}, 3 \mathrm{H}), 3.25(\mathrm{~s}, 1 \mathrm{H}), 4.26(\mathrm{q}, J=7.2 \mathrm{~Hz}, 2 \mathrm{H}), 5.91(\mathrm{~d}, J=3.0 \mathrm{~Hz}, 1 \mathrm{H})$, 7.31-7.44 (m, 5H); ${ }^{13} \mathrm{C}$ NMR $\left(\mathrm{CDCl}_{3}\right) \delta 14.41,61.15,68.64,125.67,128.26,128.70,138.81,166.40$. (For racemic 3a, see: Pellicciari, R.; Natalini, B.; Cecchetti, S.; Fringuelli, R. J. Chem. Soc. Perkin Trans. 1, 1985, 493; Jiang, N.; Wang, J. Tetrahedron Lett. 2002, 43, 1285.)

Ethyl 2-Diazo-3-phenyl-3-acetoxypropanoate $8 a$. $72 \%$ ee. $[\alpha]_{\mathrm{D}}{ }^{20}=-67.8\left(\mathrm{c} 0.95, \mathrm{CH}_{2} \mathrm{Cl}_{2}\right)$; IR 2981, 2101, 1751, 1701, 1372, $1250 \mathrm{~cm}^{-1} ;{ }^{1} \mathrm{H}$ NMR $\left(\mathrm{CDCl}_{3}\right) \delta 1.23(\mathrm{t}, J=7.2 \mathrm{~Hz}, 3 \mathrm{H}), 2.09$ (s, 3H), 4.23 (q, $J$ $=7.2 \mathrm{~Hz}, 2 \mathrm{H}), 6.85(\mathrm{~s}, 1 \mathrm{H}), 7.28-7.38(\mathrm{~m}, 5 \mathrm{H}) ;{ }^{13} \mathrm{C} \mathrm{NMR}\left(\mathrm{CDCl}_{3}\right) \delta 13.86,20.26,60.74,69.78,125.19$, 128.08, 128.40, 136.04, 164.13, 168.88; MS m/z (EI) $234\left[\left(\mathrm{M}-\mathrm{N}_{2}\right)^{+}, 0.1\right], 192$ (70), 175 (3), 146 (25), 129 (17), 118 (100), 105 (60.5), 90 (45). Anal. calcd for $\mathrm{C}_{13} \mathrm{H}_{14} \mathrm{~N}_{2} \mathrm{O}_{4}$ : C, 59.54; H, 5.38; N, 10.68. Found: C, 59.53; H, 5.44; N, 11.03 . 
Ethyl 2-Diazo-3-(3-trifluoromethyl)phenyl-3-acetoxyproponate 8b. $65 \%$ ee; $[\alpha]_{\mathrm{D}}{ }^{20}=-9.8$ (c 0.7, $\left.\mathrm{CH}_{2} \mathrm{Cl}_{2}\right)$; IR 2987, 2104, 1752, 1700, 1331, 1222, 1174, $1126 \mathrm{~cm}^{-1} ;{ }^{1} \mathrm{H}$ NMR $\left(\mathrm{CDCl}_{3}\right) \delta 1.27$ (t, $J=7.2$ $\mathrm{Hz}, 3 \mathrm{H}), 2.18(\mathrm{~s}, 3 \mathrm{H}), 4.26(\mathrm{q}, J=7.2 \mathrm{~Hz}, 2 \mathrm{H}), 6.87(\mathrm{~s}, 1 \mathrm{H}), 7.52-7.63(\mathrm{~m}, 4 \mathrm{H}) ;{ }^{13} \mathrm{C}$ NMR $\left(\mathrm{CDCl}_{3}\right) \delta$ $14.15,20.64,61.29,69.62,121.87,122.47,125.28,129.06,129.35,130.48-131.77$ (q, $J=129 \mathrm{~Hz}$ ), 137.65, 164.28, 169.33; MS m/z (EI) 302 [(M-N $\left.)^{+}, 0.8\right], 260$ (67), 215 (22), 186 (100), 173 (47), 158 (26), 145 (21). Anal. calcd for $\mathrm{C}_{14} \mathrm{H}_{13} \mathrm{~F}_{3} \mathrm{~N}_{2} \mathrm{O}_{4}$ : C, 50.91; H, 3.97; N, 8.48. Found: C, 50.69; H, 3.98; N, 8.71.

Ethyl 2-Diazo-3-chlorophenyl-3-acetoxyproponate 8c. $72 \%$ ee; $[\alpha]_{\mathrm{D}}{ }^{20}=+57.8\left(\mathrm{c} 0.55, \mathrm{CH}_{2} \mathrm{Cl}_{2}\right)$; IR 2980, 2102, 1750, 1700, 1218, 1018, $756 \mathrm{~cm}^{-1} ;{ }^{1} \mathrm{H} \mathrm{NMR}\left(\mathrm{CDCl}_{3}\right) \delta 1.27$ (t, $\left.J=7.2 \mathrm{~Hz}, 3 \mathrm{H}\right), 2.15(\mathrm{~s}, 1 \mathrm{H})$, $4.24(\mathrm{q}, J=7.2 \mathrm{~Hz}, 2 \mathrm{H}), 6.78(\mathrm{~s}, 1 \mathrm{H}), 7.28-7.37(\mathrm{~m}, 4 \mathrm{H}) ;{ }^{13} \mathrm{C} \mathrm{NMR}\left(\mathrm{CDCl}_{3}\right) \delta 14.22,20.72,61.19,69.57$,

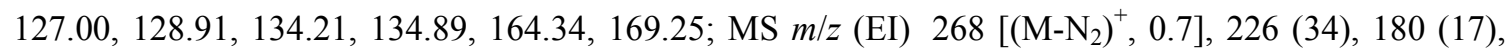
152 (63), 139 (31), 91 (51), 43 (100), 29 (72). Anal. calcd for $\mathrm{C}_{13} \mathrm{H}_{13} \mathrm{ClN}_{2} \mathrm{O}_{4}$ : C, 52.62; H, 4.42; N, 9.44. Found: C, 52.66; H, 4.54; N, 9.37.

Ethyl 2-Diazo-3-bromophenyl-3-acetoxyproponate 8d. $78 \%$ ee; $[\alpha]_{\mathrm{D}}{ }^{20}=-45.1\left(\mathrm{c} 0.75, \mathrm{CH}_{2} \mathrm{Cl}_{2}\right)$; IR 2983, 2102, 1750, 1699, 1374, 1221, 1018, $750 \mathrm{~cm}^{-1} ;{ }^{1} \mathrm{H} \mathrm{NMR}\left(\mathrm{CDCl}_{3}\right) \delta 1.28$ (t, $\left.J=7.2 \mathrm{~Hz}, 3 \mathrm{H}\right), 2.16$ $(3 \mathrm{H}, \mathrm{s}), 4.26$ (q, $J=7.2 \mathrm{~Hz}, 2 \mathrm{H}), 6.77(\mathrm{~s}, 1 \mathrm{H}), 7.25-7.50(4 \mathrm{H}, \mathrm{m}) ;{ }^{13} \mathrm{C} \mathrm{NMR}\left(\mathrm{CDCl}_{3}\right) \delta 14.33,20.85$, $61.35,69.45,122.89,124.30,128.74,130.40,131.65,138.76,164.41,169.31 ; \mathrm{MS} m / z$ (EI): 312 $\left[\left(\mathrm{M}-\mathrm{N}_{2}\right)^{+}, 0.1\right], 270$ (66), $226(25), 196$ (100), 183 (52). Anal. calcd for $\mathrm{C}_{13} \mathrm{H}_{13} \mathrm{BrN}_{2} \mathrm{O}_{4} \mathrm{C}, 45.77 ; \mathrm{H}, 3.84$; N, 8.21. Found: C, 46.34; H, 3.88; N, 7.88 .

Ethyl 2-Diazo-5-phenyl-3-hydroxypent-4-enoate 3e. $79 \%$ ee; $[\alpha]_{\mathrm{D}}{ }^{20}=-11\left(\mathrm{c} 0.4, \mathrm{CH}_{2} \mathrm{Cl}_{2}\right)$; IR 3435, 2983, 2098, 1683, 1378, $1336 \mathrm{~cm}^{-1} ;{ }^{1} \mathrm{H}$ NMR $\left(\mathrm{CDCl}_{3}\right) \delta 1.28(\mathrm{t}, J=7.2 \mathrm{~Hz}, 3 \mathrm{H}), 3.24$ (s, 1H), 4.26 (q, $J$ $=7.2 \mathrm{~Hz}, 2 \mathrm{H}), 5.42-5.46(\mathrm{~m}, 1 \mathrm{H}), 6.25(\mathrm{dd}, J=5.4,15.9 \mathrm{~Hz}, 1 \mathrm{H}), 6.79(\mathrm{~d}, J=15.9 \mathrm{~Hz}, 1 \mathrm{H}), 7.23-7.41$ $(\mathrm{m}, 5 \mathrm{H}) ;{ }^{13} \mathrm{C} \mathrm{NMR}\left(\mathrm{CDCl}_{3}\right) \delta 14.39,61.13,66.97,114.70,125.66,126.63,128.06,128.57,132.05$, 135.88, 166.15; MS m/z (EI) 218 [(M-N $\left.)^{+}, 11\right], 171$ (30), 144 (33), 131 (71), 115 (64), 103 (48), 69 (76), 57 (100). Anal. calcd for $\mathrm{C}_{13} \mathrm{H}_{14} \mathrm{~N}_{2} \mathrm{O}_{3}: \mathrm{C}, 63.40 ; \mathrm{H}, 5.73 ; \mathrm{N}, 11.38$. Found: C, 63.45; H, 5.77; N, 11.15.

Ethyl 2-Diazo-3-[2-(6-methylpyridyl)]-3-hydroxyproponate $3 f .53 \%$ ee; $[\alpha]_{\mathrm{D}}^{20}=-10$ (c 0.3 , $\left.\mathrm{CH}_{2} \mathrm{Cl}_{2}\right)$; IR 3413, 2982, 2098, 1691, 1588, $1459 \mathrm{~cm}^{-1} ;{ }^{1} \mathrm{H} \mathrm{NMR}\left(\mathrm{CDCl}_{3}\right) \delta 1.28(\mathrm{t}, J=7.2 \mathrm{~Hz}, 3 \mathrm{H}), 2.55$ (s, 3H), $4.26(\mathrm{q}, J=7.2 \mathrm{~Hz}, 2 \mathrm{H}), 5.40(\mathrm{~s}, 1 \mathrm{H}), 5.77(1 \mathrm{H}, \mathrm{s}), 7.10(\mathrm{~d}, J=7.8,1 \mathrm{H}), 7.23(\mathrm{~d}, J=7.8,1 \mathrm{H})$, 7.60-7.65 (t, $J=7.8,7.8 \mathrm{~Hz}, 1 \mathrm{H}) ;{ }^{13} \mathrm{C} \mathrm{NMR}\left(\mathrm{CDCl}_{3}\right) \delta 14.22,23.85,60.71,66.75,117.65,122.52,117.65$,

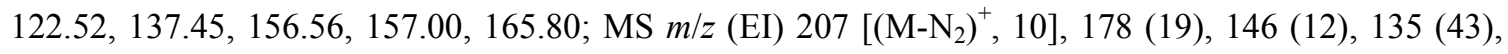
120 (18), 93 (100), 84 (98). Anal. calcd for $\mathrm{C}_{11} \mathrm{H}_{13} \mathrm{~N}_{3} \mathrm{O}_{3}: \mathrm{C}, 56.16 ; \mathrm{H}, 5.57 ; \mathrm{N}, 17.86$. Found: $\mathrm{C}, 56.30 ; \mathrm{H}$, $5.65 ; \mathrm{N}, 17.43$.

Ethyl 2-Diazo-3-(2-Furyl)-3-hydroxyproponate 3g. $86 \%$ ee; $[\alpha]_{\mathrm{D}}{ }^{20}=-15\left(\mathrm{c} 0.4, \mathrm{CH}_{2} \mathrm{Cl}_{2}\right)$; IR 3415, 2986, 2104, 1682, 1380, $1291 \mathrm{~cm}^{-1} ;{ }^{1} \mathrm{H}$ NMR (CDCl $) \delta 1.27$ (t, $\left.J=7.2 \mathrm{~Hz}, 3 \mathrm{H}\right), 3.98$ (s, 1H), 4.23 (q, $J$ $=7.2 \mathrm{~Hz}, 2 \mathrm{H}), 5.80(\mathrm{~s}, 1 \mathrm{H}), 6.34-6.37(\mathrm{~m}, 2 \mathrm{H}), 7.39(\mathrm{~s}, 1 \mathrm{H}) ;{ }^{13} \mathrm{C} \mathrm{NMR}\left(\mathrm{CDCl}_{3}\right) \delta 14.21,61.12,63.10$, 
107.19, 110.21, 142.56, 151.91, 165.88; MS $m / z$ (EI) $210\left(\mathrm{M}^{+}, 2\right), 182\left[\left(\mathrm{M}-\mathrm{N}_{2}\right)^{+}, 4\right], 154(25), 136(84)$, 108 (41), 95 (93); Anal. calcd for $\mathrm{C}_{9} \mathrm{H}_{10} \mathrm{~N}_{2} \mathrm{O}_{4}$ : C, 51.43; H, 4.80; N, 13.33. Found: C, 51.83; H, 5.06; N, 12.97.

Ethyl 2-Diazo-3-hydroxyhexanoate $3 \boldsymbol{h} .57 \%$ ee; $[\alpha]_{\mathrm{D}}{ }^{20}=+9.3\left(\mathrm{c} 0.3, \mathrm{CH}_{2} \mathrm{Cl}_{2}\right)$; IR 3410, 2967, 2095, 1688, 1379, $1296 \mathrm{~cm}^{-1} ;{ }^{1} \mathrm{H}$ NMR $\left(\mathrm{CDCl}_{3}\right) \delta 0.95(\mathrm{t}, J=7.2 \mathrm{~Hz}, 3 \mathrm{H}), 1.29(\mathrm{t}, J=6.9 \mathrm{~Hz}, 3 \mathrm{H}), 1.33-1.73$ (m, 4H), 3.55 (s, 1H), 4.20-4.27 (q, $J=7.2 \mathrm{~Hz}, 2 \mathrm{H}), 4.64-4.70(\mathrm{~m}, 1 \mathrm{H}) ;{ }^{13} \mathrm{C} \mathrm{NMR}\left(\mathrm{CDCl}_{3}\right) \delta 13.49,14.22$,

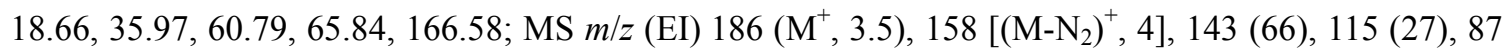
(60), 71(44), 55(95); Anal. calcd for $\mathrm{C}_{8} \mathrm{H}_{14} \mathrm{~N}_{2} \mathrm{O}_{3}$ : C, 51.60; H, 7.58; N, 15.04. Found: C, 51.32; H, 7.44; $\mathrm{N}, 15.15$.

Determination of the absolute configuration of $\mathbf{3 a}$. The absolute configuration of $\mathbf{3 a}$ was determined to be $R$ by converting it to a known compound through hydrogenation. The hydrogenation of 3a (87 \% ee) following the literature procedure (Pellicciari, R.; Natalini, B.; Cecchetti, S.; Fringuelli, R. J. Chem. Soc. Perkin Trans. 1, 1985, 493) resulted in partial racemization, but it was possible to determine the absolute configuration by comparing the sign of optical rotation with a known compound.

3a (35 mg, $0.16 \mathrm{mmol}, 87 \%$ ee) was dissolved $\mathrm{MeOH}(2 \mathrm{~mL})$, and $10 \% \mathrm{Pd} / \mathrm{C}(15 \mathrm{mg})$ was added. The mixture was hydrogenated under $1 \mathrm{~atm} \mathrm{H}_{2}$ for $1 \mathrm{~h}$, and then the mixture was filtered. The filtrate was removed with rotvap, and the residue was purified by silica gel column (petroleum ether : acetone $=$ $4: 1)$ to give 3-hydroxy-3-phenylpropionate (20 mg, $64 \%)$. $[\alpha]_{\mathrm{D}}{ }^{20}=-2.3\left(\mathrm{c} 0.3, \mathrm{CHCl}_{3}\right)$, literature data for (S)-3-hydroxy-3-phenylpropionate: $[\alpha]_{\mathrm{D}}{ }^{20}=-44.8$ (c 1.6, $\mathrm{CHCl}_{3}$ ) (Cabon, O.; Buisson, D.; Larcheveque, M.; Azerad, R. Tetrahedron: Asymmetry 1995, 6, 2199-2210).

Oxidation of Ethyl 2-Diazo-3-phenyl-3-acetoxypropanoate $8 \boldsymbol{a}$. $\mathrm{NaHCO}_{3}(332 \mathrm{mg}, 4 \mathrm{mmol})$ was added to the solution of $\mathrm{H}_{2} \mathrm{O}(1.5 \mathrm{~mL})$ and acetone $(1 \mathrm{~mL})$ and the mixture was cooled by ice-bath. Oxone ${ }^{\circledR}(625$ $\mathrm{mg} 1.0 \mathrm{mmol}$ ) was then added. To this mixture, $8 \mathbf{a}\left(52 \mathrm{mg}, 0.2 \mathrm{mmol}, 72 \%\right.$ ee) in $\mathrm{CH}_{2} \mathrm{Cl}_{2}(1.5 \mathrm{~mL})$ was added dropwise in 15 min under nitrogen atmosphere. Ice-bath was then removed and the temperature was raised to room temperature. The yellow color was disappeared after about $1.5 \mathrm{~h}$, and TLC check indicated that the starting material had disappeared. The mixture was extracted by $\mathrm{CH}_{2} \mathrm{Cl}_{2}$ twice. The combined extracts were dried over anhydrous $\mathrm{Na}_{2} \mathrm{SO}_{4}$. The solvent was removed by rotvap, and the residue was purified by silica gel column (petroleum ether : acetone $=4: 1$ ) to give ethyl 2-Oxo-3-phenyl-3-acetoxypropanoate 9 (45 mg, $90 \%, 73 \%$ ee). [ $\alpha]_{\mathrm{D}}{ }^{20}=+145$ (c $0.65, \mathrm{CH}_{2} \mathrm{Cl}_{2}$ ); IR 3477, 2987, 1739, 1373, 1230, $1033 \mathrm{~cm}^{-1} ;{ }^{1} \mathrm{H}$ NMR $\left(\mathrm{CDCl}_{3}\right) \delta 1.27(\mathrm{t}, J=7.2 \mathrm{~Hz}, 3 \mathrm{H}), 2.19(\mathrm{~s}, 3 \mathrm{H}), 4.26$ (q, $J=7.2 \mathrm{~Hz}, 2 \mathrm{H}), 6.66(\mathrm{~s}, 1 \mathrm{H}), 7.38-7.48(\mathrm{~m}, 5 \mathrm{H}) ;{ }^{13} \mathrm{C} \mathrm{NMR}\left(\mathrm{CDCl}_{3}\right): 13.79,20.46,62.75,77.76$, 128.90, 129.05, 129.74, 130.83, 159.25, 170.20, 186.66; MS $m / z$ (EI) $250\left(\mathrm{M}^{+}, 0.1\right), 208$ (0.8), 149 (48), 107 (98), 105 (10), 90 (5), 79 (16), 43 (100).

Reduction of ethyl 2-Oxo-3-phenyl-3-acetoxypropanoate $9.9(20 \mathrm{mg}, 0.08 \mathrm{mmol})$ was dissolved in EtOH $(2 \mathrm{~mL})$ and the solution was cooled by dry ice- $\mathrm{ClCH}_{2} \mathrm{CH}_{2} \mathrm{Cl}$ bath $\left(-35^{\circ} \mathrm{C}\right) . \mathrm{NaBH}_{4}(3 \mathrm{mg}, 0.08$ mmol) was added and the solution was stirred for $1 \mathrm{~h}$. Saturated aqueous $\mathrm{NH}_{4} \mathrm{Cl}$ was then added and the 
mixture was extracted with $\mathrm{CH}_{2} \mathrm{Cl}_{2}$ for three times. The combined extracts were dried over anhydrous $\mathrm{Na}_{2} \mathrm{SO}_{4}$. Solvent was removed and the residue was purified by silica gel column (petroleum ether : acetone $=4: 1)$ to give an oil $(20 \mathrm{mg}, 99 \%)$. Inspection of the product suggested that it was a diastereomeric mixture of syn- and anti- ethyl 2-hydroxy-3-phenyl-3-acetoxyproponates with a ratio of $12: 1$. The major isomer was confirmed to be anti by converting it to diol $\mathbf{1 1}$ and by comparison with the ${ }^{1} \mathrm{H}$ NMR spectra of a known compound (vide infra). ${ }^{1} \mathrm{H}$ NMR $\left(\mathrm{CDCl}_{3}\right)$ for major anti isomer 10: $\delta 1.27$ (t, $J=7.2 \mathrm{~Hz}, 3 \mathrm{H}), 2.15$ (s, 3H), 2.88 (d, $J=7.2 \mathrm{~Hz}, 1 \mathrm{H}), 4.22$ (t, $J=7.2 \mathrm{~Hz}, 2 \mathrm{H}), 4.61$ (dd, $J=3.6,7.2$ $\mathrm{Hz}, 1 \mathrm{H}), 6.07(\mathrm{~d}, J=3.9 \mathrm{~Hz}, 1 \mathrm{H}), 7.33-7.40(\mathrm{~m}, 5 \mathrm{H}) .{ }^{13} \mathrm{C} \mathrm{NMR}\left(\mathrm{CDCl}_{3}\right)$ for major anti isomer 10: $\delta 14.07,21.05,62.21,72.90,76.02,127.32,128.28,128.66,134.84,169.92,171.38$.

Hydrolysis of ethyl 2-hydroxy-3-phenyl-3-acetoxyproponate with catalytic $\mathrm{K}_{2} \mathrm{CO}_{3}$. A literature procedure (Desai, S. B.; Argade, N. P.; Ganesh, K. N. J. Org. Chem. 1996, 61, 6730.) was followed. The mixture of syn- and anti- ethyl 2-hydroxy-3-phenyl-3-acetoxyproponates $(30 \mathrm{mg}, 0.12 \mathrm{mmol}$ ) was dissolved in anhydrous EtOH $(2 \mathrm{~mL})$, and then $\mathrm{K}_{2} \mathrm{CO}_{3}(1 \mathrm{mg})$ was added. The solution was stirred for $4 \mathrm{~h}$ at room temp. under $\mathrm{N}_{2}$. The solution was filtered and the filtrate was evaporated in vacuum. The residue was purified with silica gel column (petroleum ether : acetone $=4: 1)$ to give of $\mathbf{1 1}(20 \mathrm{mg}, 84 \%) .{ }^{1} \mathrm{H}$ NMR $\left(\mathrm{CDCl}_{3}\right) \delta 1.16(\mathrm{t}, J=7.2 \mathrm{~Hz}, 3 \mathrm{H}), 2.94(\mathrm{~d}, J=6.0 \mathrm{~Hz}, 1 \mathrm{H}), 3.00(\mathrm{~d}, J=6.6 \mathrm{~Hz}, 1 \mathrm{H}), 4.10-4.17(\mathrm{t}, J=$ $7.2 \mathrm{~Hz}, 2 \mathrm{H}), 4.49(\mathrm{dd}, J=4.2,6 \mathrm{~Hz}, 1 \mathrm{H}), 5.01-5.04(\mathrm{~m}, 1 \mathrm{H}), 7.30-7.43(\mathrm{~m}, 5 \mathrm{H})$.

This product was different from the sample of syn- ethyl 2,3-dihydroxy-3-phenylproponate, which was prepared from the dihydroxylation of trans- ethyl cinnamate with $\mathrm{KMnO}_{4}{ }^{1}{ }^{\mathrm{H}} \mathrm{NMR}\left(\mathrm{CDCl}_{3}\right) \delta 1.27$ $(\mathrm{t}, J=6.9 \mathrm{~Hz}, 3 \mathrm{H}), 2.77$ (d, $J=7.2 \mathrm{~Hz}, 1 \mathrm{H}), 3.13(\mathrm{~d}, J=5.7 \mathrm{~Hz}, 1 \mathrm{H}), 4.26(\mathrm{t}, J=7.2 \mathrm{~Hz}, 2 \mathrm{H}), 4.37$ (dd, $J$ $=3.0,5.4 \mathrm{~Hz}, 1 \mathrm{H}), 5.00(\mathrm{~s}, 1 \mathrm{H}), 7.29-7.47(\mathrm{~m}, 5 \mathrm{H})$. 
Chiralcel OJ; Flow $=0.8 \mathrm{~mL} / \mathrm{min}$; hexane $/$ iso-propanol $=96: 4$

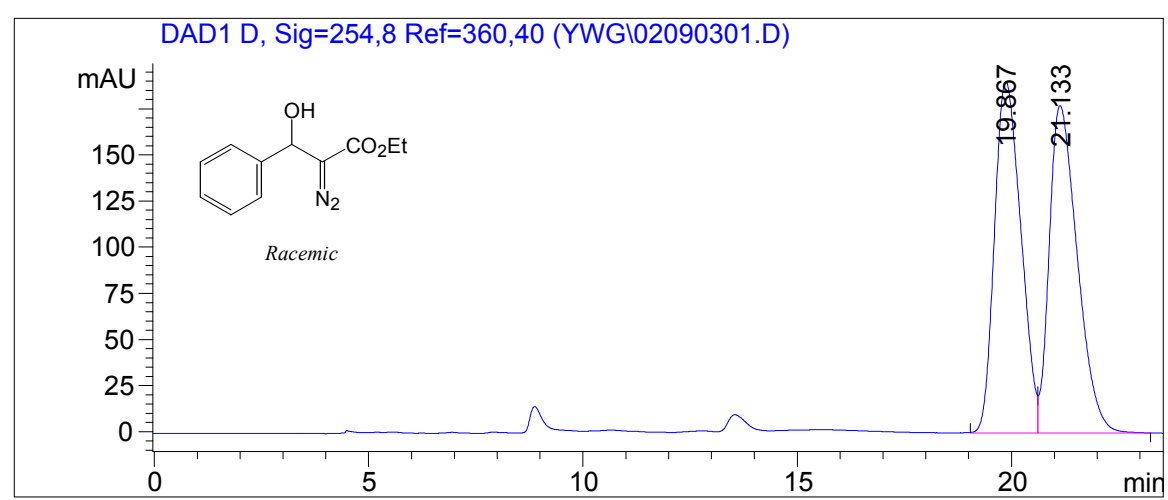

Table 1, entry 7

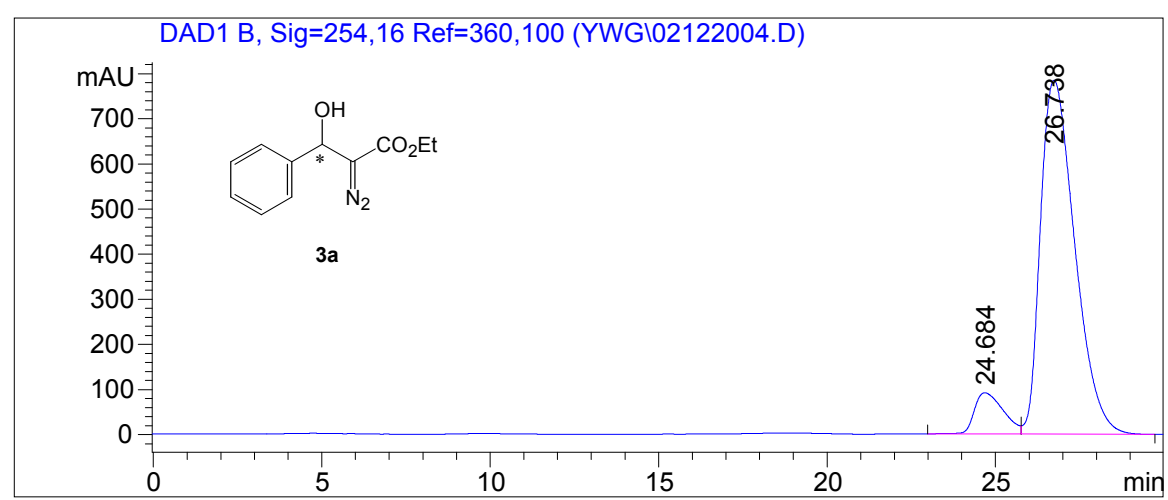

Table 1 , entry 8 (Table 2 , entry 1 )

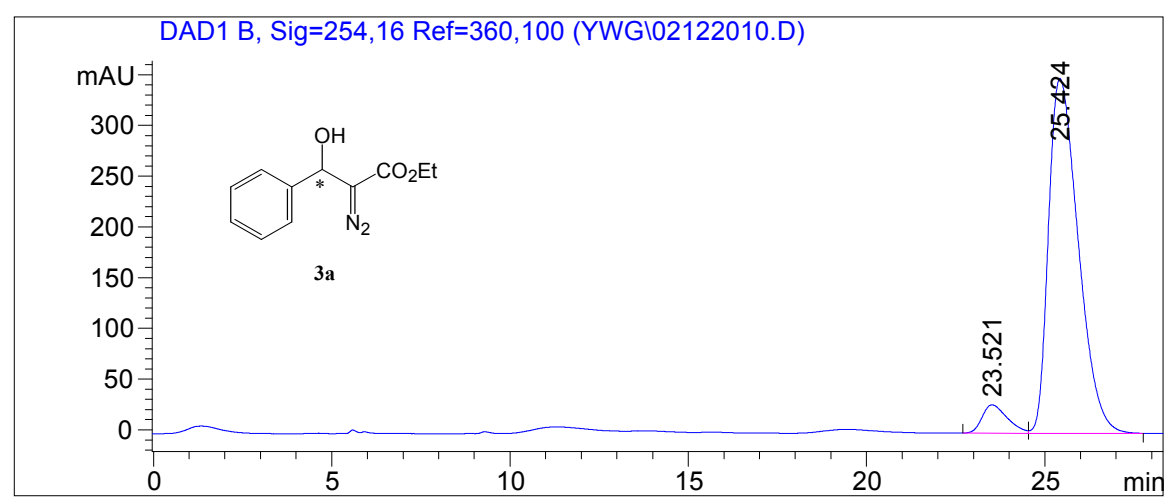


Chiralcel OJ, Flow $=1.0 \mathrm{~mL} / \mathrm{min}$, hexane $/$ iso -propanol $=99.7: 0.3$

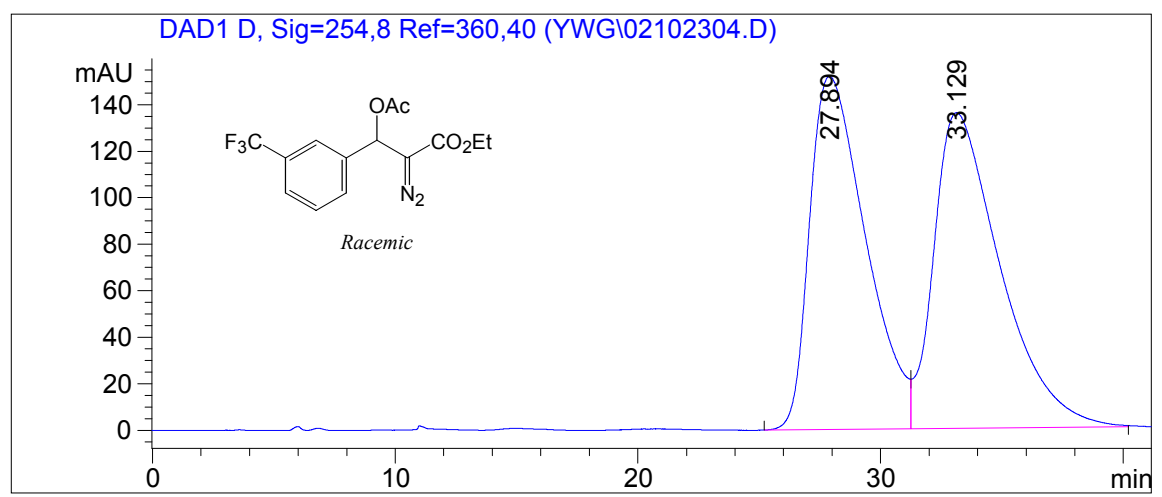

Table 2, entry 2 .

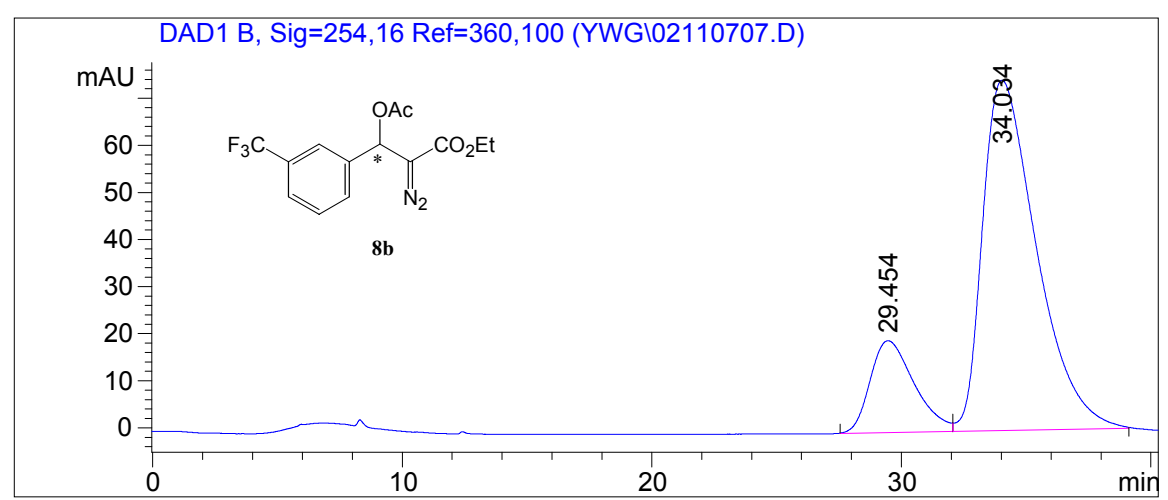

Chiralcel OJ, Flow $=0.8 \mathrm{~mL} / \mathrm{min}$, hexane $/$ iso-propanol $=98: 2$

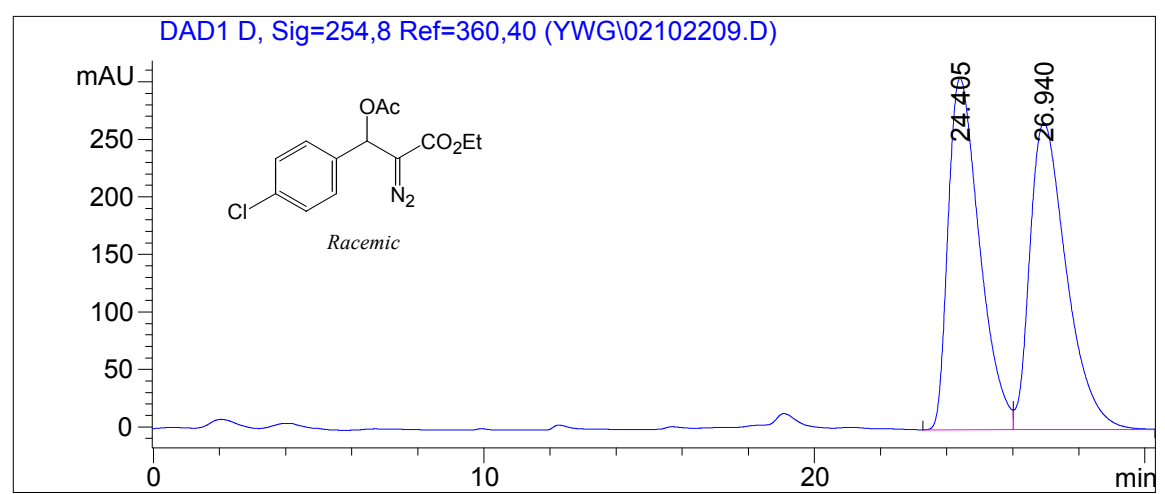


Table 2, entry 3

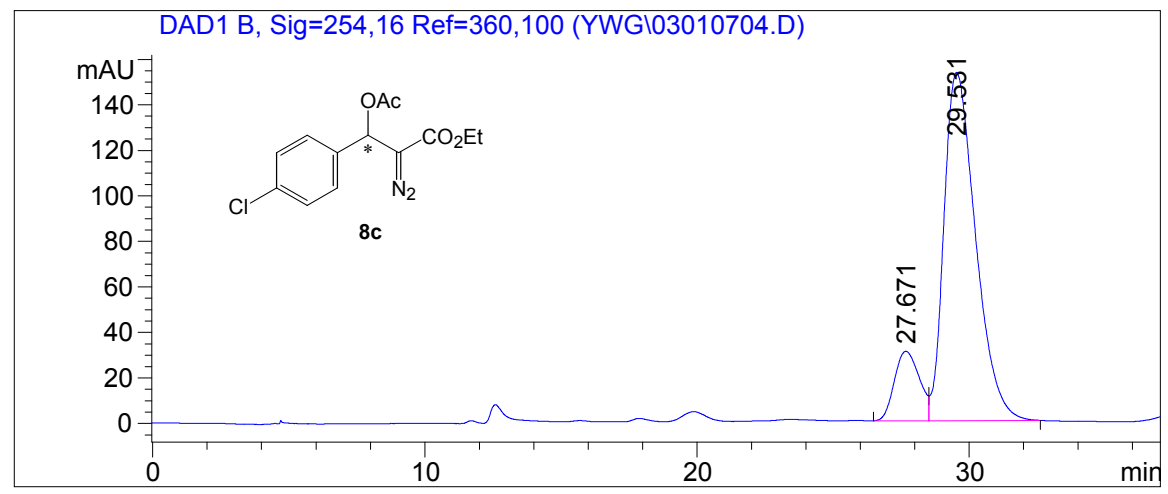

Chiralcel OJ, Flow $=0.5 \mathrm{~mL} / \mathrm{min}$, hexane $/$ iso-propanol $=95: 5$

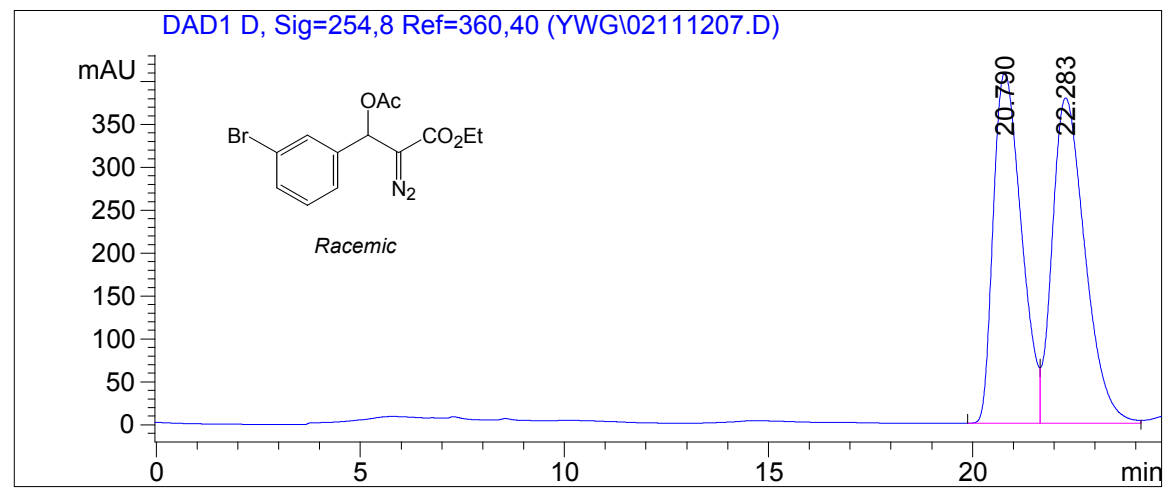

Table 2, entry 4.

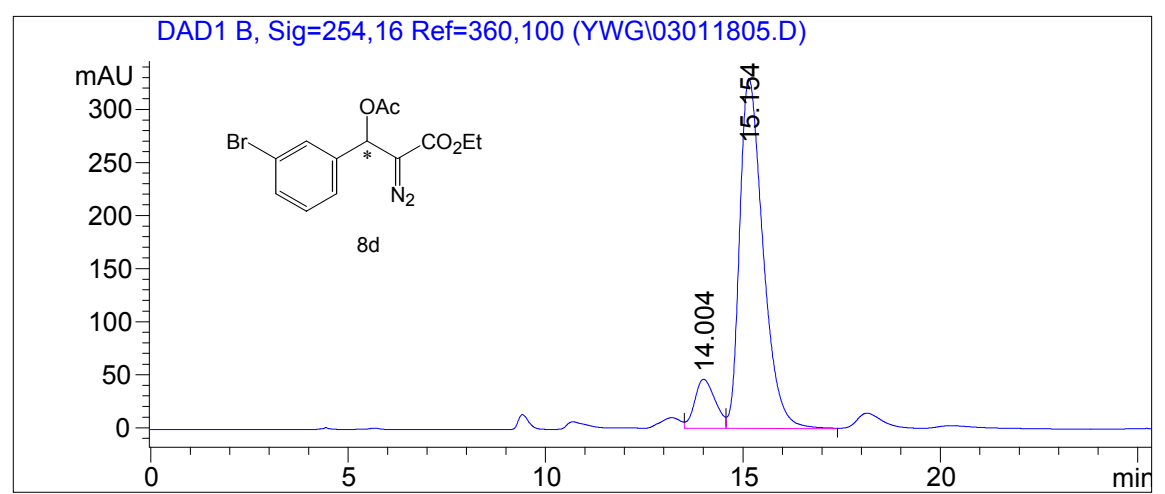


Chiralcel OJ, Flow $=0.8 \mathrm{~mL} / \mathrm{min}$, hexane $/$ iso-propanol $=90: 10$

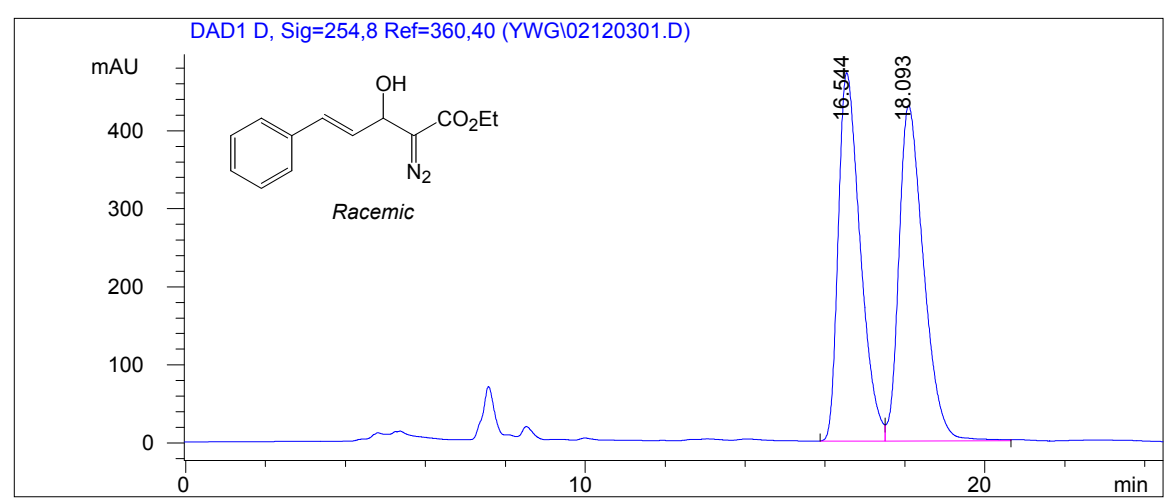

Chiralcel OJ, Flow $=0.8 \mathrm{~mL} / \mathrm{min}$, hexane/iso-propanol $=93: 7$; Table 2, entry 5 .

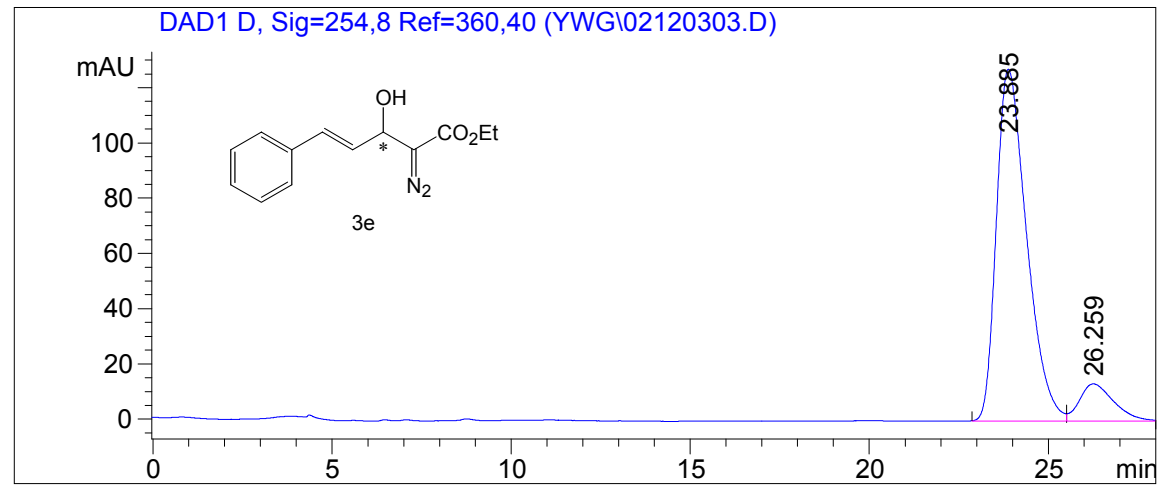

Chiralcel OJ, Flow $=0.8 \mathrm{~mL} / \mathrm{min}$, hexane $/$ iso-propanol $=90: 10$

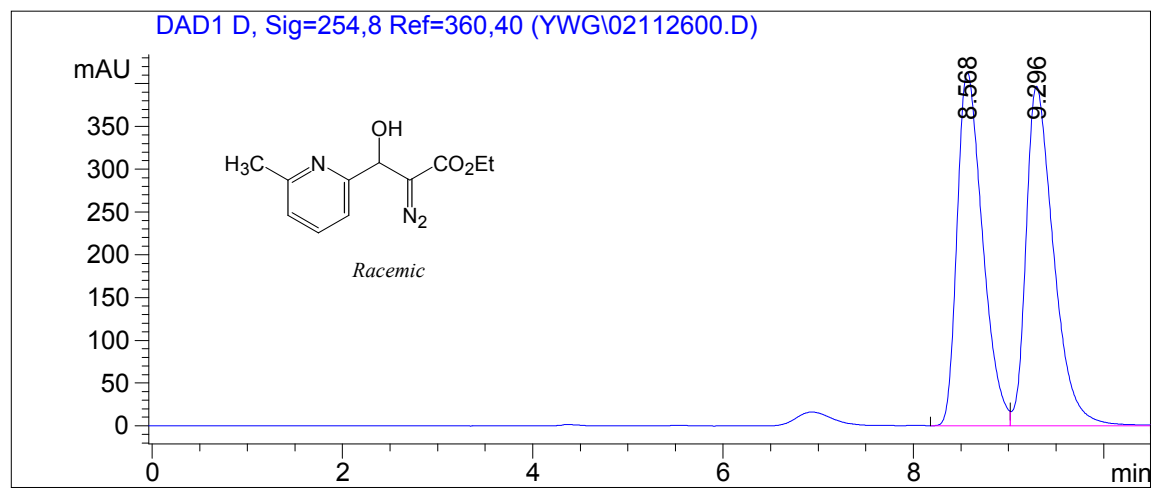


Table 2, entry 6

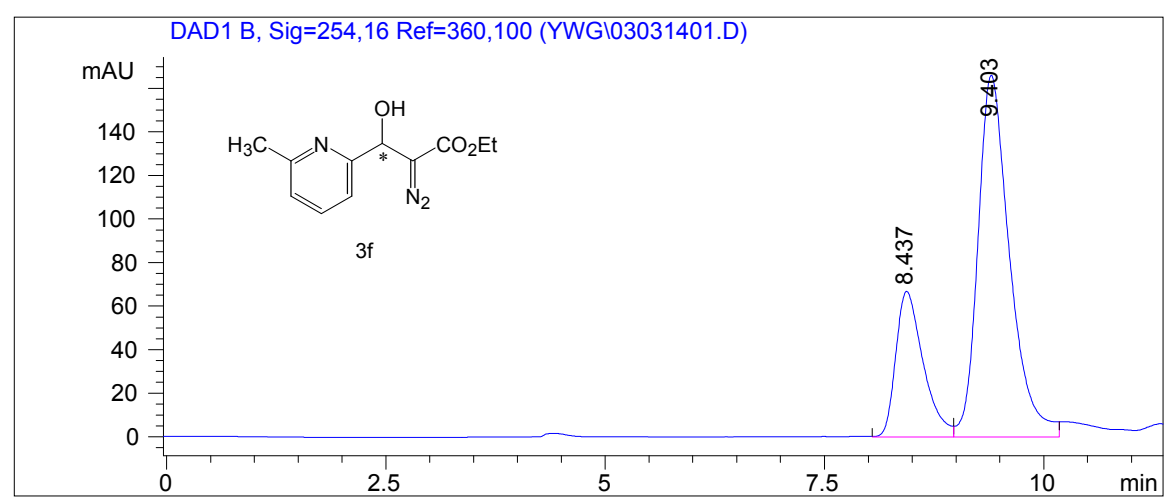

Chiralcel OJ, Flow $=0.8 \mathrm{~mL} / \mathrm{min}$, hexane $/$ iso-propanol $=90: 10$

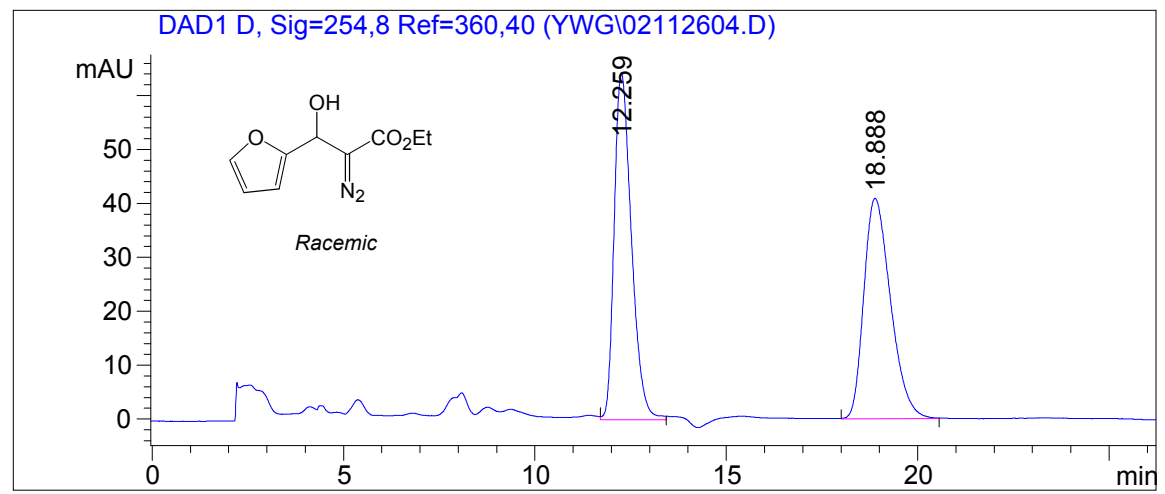

Table 2, entry 7

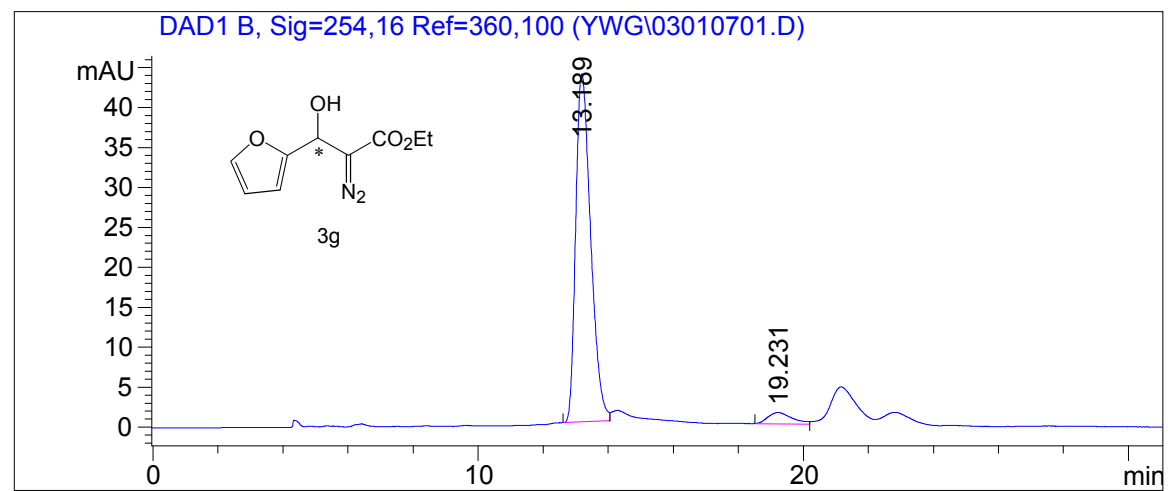


Chiralcel OJ, Flow $=0.8 \mathrm{~mL} / \mathrm{min}$, hexane $/$ iso -propanol $=99.5: 0.5$

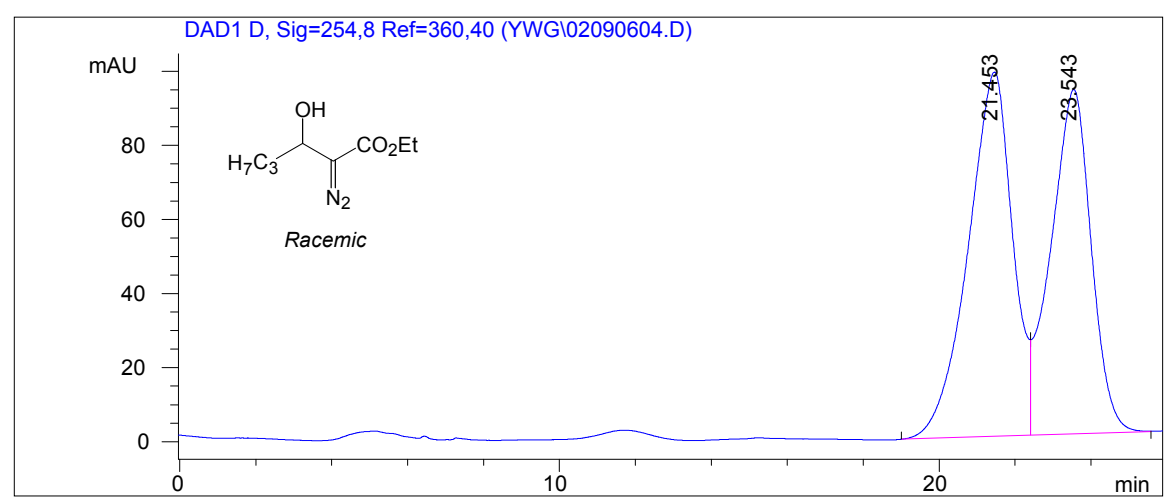

Table 2, entry 8

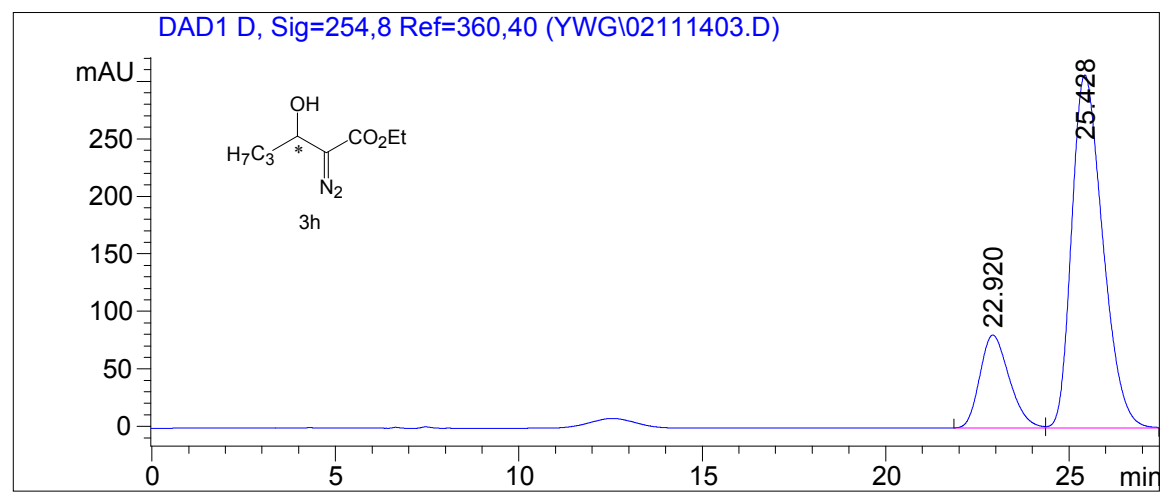

Chiralcel OJ, Flow $=0.8 \mathrm{~mL} / \mathrm{min}$, hexane $/$ iso-propanol $=95: 5$

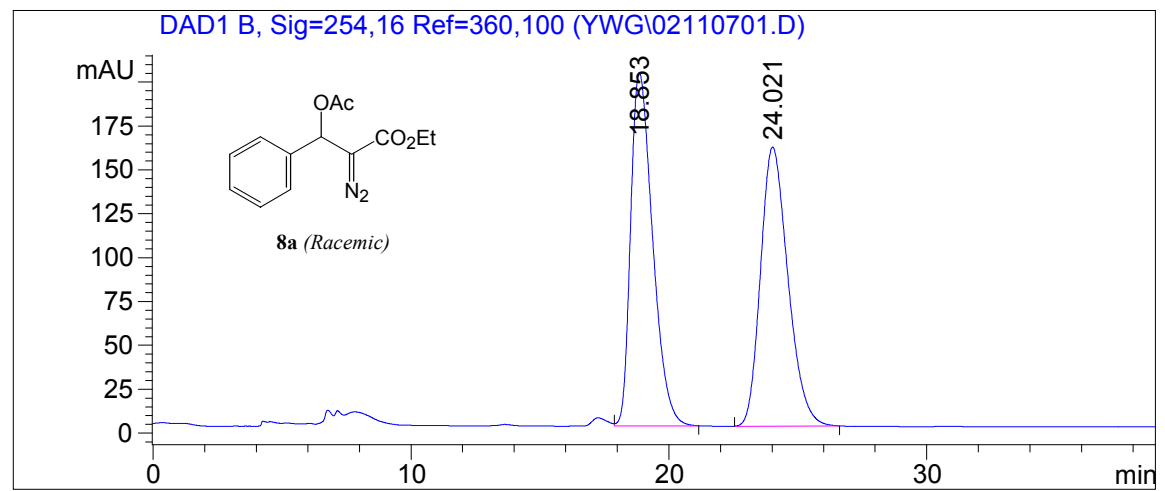




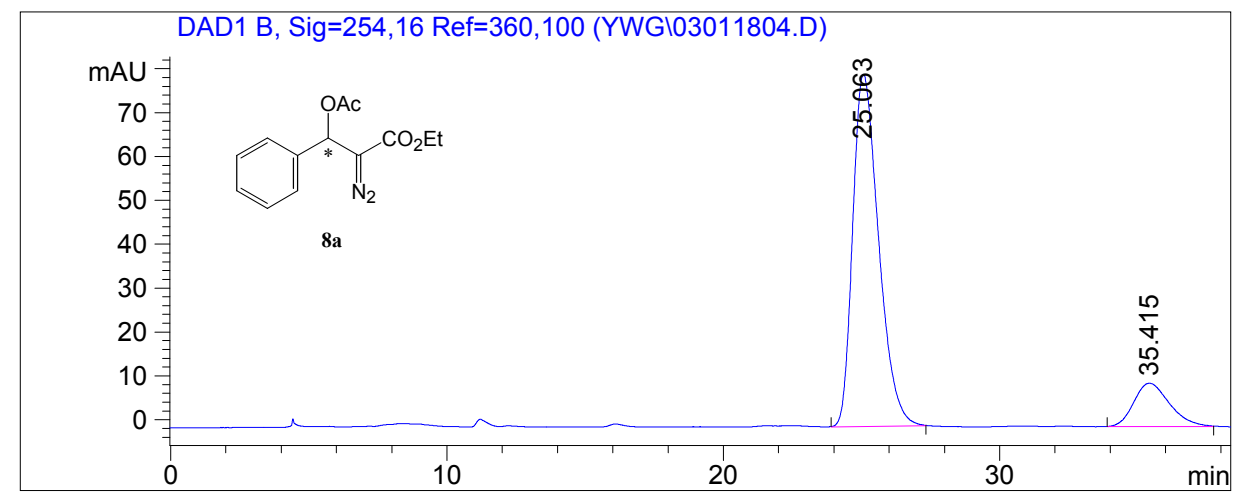

